\title{
A Note on Summation Formulas of Powers of Roots
}

\author{
By Daniel C. Fielder
}

Introduction. Through application of a method due to Newton [1], it is possible to find solutions for the sum $S_{b}$ of the $b$ th powers of the $n$ roots of $f(x)$, where $f(x)$ is an integral rational function of $n$th degree in $x$. As anyone who has used this method for large $n$ and $b$ can attest, the procedure involves tedious and repetitive manipulations which increase rapidly in complexity as $b$ is increased.

It is shown herein that it is possible to reduce the labor of finding such algebraic sums by rejecting redundant information in a general formula for the sums and using only pertinent terms to predict the numerical and literal coefficients. Inductive reasoning is employed to arrive at a simplified method.

Conventional means for determining sums. Given the expression

$$
f(x)=a_{0} x^{n}+a_{1} x^{n-1}+\cdots a_{n-1} x+a_{n},
$$

where $f(x)$ is an integral rational function of $n$th degree in $x$, and $a_{0}, a_{1}$, etc., are the coefficients of the powers of $x$. The sum of the $b$ th powers of the roots of $f(x)$ are

$$
S_{b}=c_{1}^{b}+c_{2}^{b}+\cdots c_{n}^{b} \text {, }
$$

where $c_{1}, c_{2}$, etc., are the roots of $f(x)$. Newton's method makes use of the following tabulation to find $S_{b}$.

$$
\begin{aligned}
& 1 a_{1}+S_{1} a_{0}=0 \\
& 2 a_{2}+S_{1} a_{1}+S_{2} a_{0}=0 \\
& 3 a_{3}+S_{1} a_{2}+S_{2} a_{1}+S_{3} a_{0}=0
\end{aligned}
$$

For the case of $n=b$, to get $S_{n}$ it is necessary to go through $n$ operations, each successive one becoming increasingly involved. The only respite occurs if one is able to find a table of $S_{n}$ 's of desired scope. Unfortunately, available tables seem to end with the fourth or fifth summation. A typical listing of sums and a summary of Newton's method is given by Adams [1].

Development of a summation formula. A general summation formula is now presented. While in the interests of simplicity it is not desirable to use this formula directly to calculate $S_{n}$, it is found that a limited number of terms of this formula for a particular $S_{n}$ can be exploited advantageously to yield complete information

Received 26 November 1957. 
about the $S_{n}$. By induction, it can be shown

$$
\begin{aligned}
& S_{n}=-\frac{1}{a_{0}}\left(n a_{n}\right)+\frac{1}{a_{0}^{2}}\left[\frac{n}{2} \sum_{A=1}^{n-1}\left(a_{A} a_{n-A}\right)\right] \\
& -\frac{1}{a_{0}^{3}}\left[\frac{n}{3} \sum_{B=1}^{n-2} \sum_{A=1}^{n-1-B}\left(a_{B} a_{A} a_{n-B-A}\right)\right] \\
& +\frac{1}{a_{0}^{4}}\left[\frac{n}{4} \sum_{C=1}^{n-3} \sum_{B=1}^{n-2-C} \sum_{A=1}^{n-1-C-B}\left(a_{C} a_{B} a_{A} a_{n-C-B-A}\right)\right] \\
& \left.+\frac{(-1)^{n}}{a_{0}^{n}}[\frac{n}{n} \underbrace{\cdots}_{(n-1) \sum_{Z=1} \sum_{Y=1}^{1} \sum_{X=1}^{2-Z} \sum_{X-Z}^{3-Z}} \underbrace{\left(a_{Z} a_{Y} a_{X} \cdots a_{A} a_{n-Z-Y-X} \cdots A\right.}_{n \text { literal terms }})\right] .
\end{aligned}
$$

One needs only to consider $S_{n}$ for $n$ equal to 11 or 12 , for example, to note that the use of equation (4) results in summations of many repetitive combinations of $a$ 's. However, it can be seen that $S_{n}$ is an $n$th order integral symmetric function [2] in $a_{1}, a_{2}$, etc., with the weight of the symmetric function necessarily equal to $n$. For example, if $n$ were nine, one of the literal terms of the coefficients of $-1 / a_{0}{ }^{5}$ in equation (4) would be $a_{1}{ }^{2} a_{2}{ }^{2} a_{3}{ }^{1}$. The weight is the sum of the products of corresponding subscripts and exponents. In this case, it is $1 \times 2+2 \times 2$ $+3 \times 1=9$. A second necessary condition is that the degree of the product of the $a$ 's (exclusive of $a_{0}$ ) must equal the power of the corresponding $a_{0}$. For example, $a_{2}^{8} a_{3}{ }^{1}$ would not be found in the coefficient of any $1 / a_{0}{ }^{5}$. Thus, the coefficient of $(-1)^{k} / a_{0}{ }^{k}$ contains all the combinations of $a$ 's (exclusive of $a_{0}$ ) which simultaneously have weight of $n$ and degree of $k$.

Development of a practical summation form. To specify completely the coefficient for each $(-1)^{k} / a_{0}^{k}$ of a particular $S_{n}$, one must know (a) what the combinations of the $a$ 's are and (b) what the individual numerical coefficients of the combinations are. Obviously, equation (4), after required manipulations, yields the answer. However, if the tedious approach of equation (4) is to be avoided, the information must be obtained in a somewhat different manner from direct application of equation (4).

The answer to (a) above can be found from a slight rearrangement of a table which Euler first developed in connection with enumeration of partitions. The table is presented in extended form in Euler's "Introductio," Lib. 1, Chap. XVI, and is reproduced in Chrystal [3]. The number of literal coefficients for each $(-1)^{k} / a_{0}^{k}$ is found directly from the rearranged table which is shown herein as Table 1. A brief explanation of the mechanics of constructing the table is given at the end of this paper. The literal coefficients are found by systematically setting down combinations of the $a$ 's with weight and degree consistent with $n$ and $k$ until the number specified by Table 1 is formed. For example, if $n=11$ and $k=3$, Table 1 indicates that there are ten literal coefficients. 


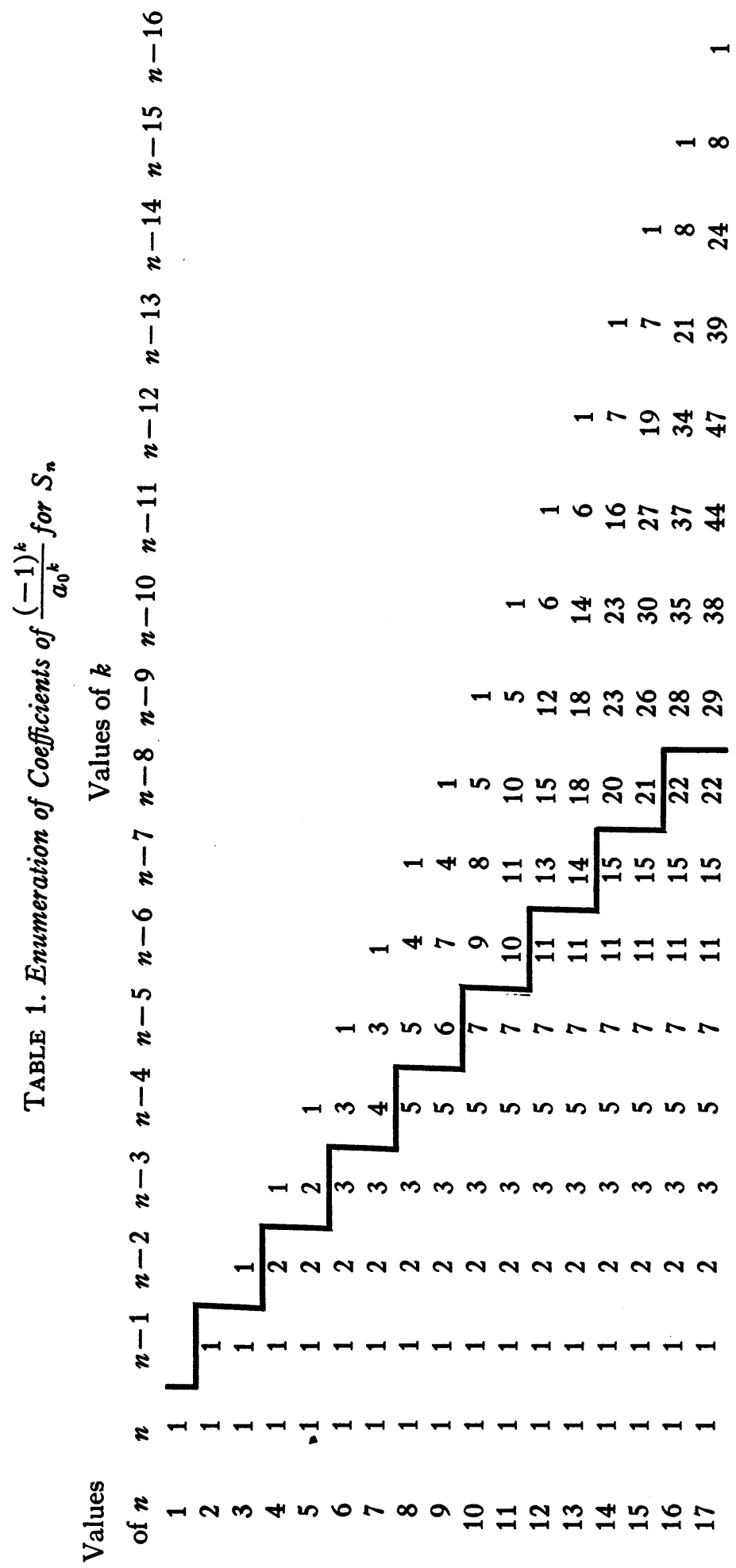


The answer to (b) above can be found from a consideration of the exponents of the $a$ 's. In the coefficient of each $(-1)^{k} / a_{0}{ }^{k}$, there always appears the combination $a_{1}{ }^{k-1} a_{n-k+1}$ which has the numerical coefficient $n$. The product $n \times(k-1)$ ! $X 1$ ! divided by the product of the factorials of the exponents of a particular

$$
\begin{aligned}
& S_{1}=-a_{1} / a_{0} \\
& \text { TABLE 2. Values of } S_{n}, n=1(1) 11 \\
& S_{2}=a_{1}^{2} / a_{0}^{2}-2 a_{2} / a_{0} \\
& S_{3}=-a_{1}^{3} / a_{0}^{3}+3 a_{1} a_{2} / a_{0}^{2}-3 a_{3} / a_{0} \text {, } \\
& S_{4}=a_{1}^{4} / a_{0}^{4}-4 a_{1}^{2} a_{2} / a_{0}^{3}+\left(4 a_{1} a_{3}+2 a_{2}^{2}\right) / a_{0}{ }^{2}-4 a_{4} / a_{0} \text {, } \\
& S_{5}=-a_{1}^{5} / a_{0}^{5}+5 a_{1}^{3} a_{2} / a_{0}^{4}-\left(5 a_{1}^{2} a_{3}+5 a_{1} a_{2}^{2}\right) / a_{0}^{3}+\left(5 a_{1} a_{4}+5 a_{2} a_{3}\right) / a_{0}^{2}-5 a_{5} / a_{0} \text {, } \\
& S_{6}=a_{1}{ }^{6} / a_{0}^{6}-6 a_{1}^{4} a_{2} / a_{0}{ }^{5}+\left(6 a_{1}^{3} a_{3}+9 a_{1}^{2} a_{2}^{2}\right) / a_{0}{ }^{4}-\left(6 a_{1}^{2} a_{4}+12 a_{1} a_{2} a_{3}+2 a_{2}^{3}\right) / a_{0}^{3} \\
& +\left(6 a_{1} a_{5}+6 a_{2} a_{4}+3 a_{3}^{2}\right) / a_{0}^{2}-6 a_{6} / a_{0} \text {, } \\
& S_{7}=-a_{1}{ }^{7} / a_{0}{ }^{7}+7 a_{1}{ }^{5} a_{2} / a_{0}{ }^{6}-\left(7 a_{1}{ }^{4} a_{3}+14 a_{1}{ }^{3} a_{2}{ }^{2}\right) / a_{0}{ }^{5} \\
& +\left(7 a_{1}^{3} a_{4}+21 a_{1}{ }^{2} a_{2} a_{3}+7 a_{1} a_{2}^{3}\right) / a_{0}{ }^{4}-\left(7 a^{2} a_{5}+14 a_{1} a_{2} a_{4}+7 a_{2}^{2} a_{3}+7 a_{1} a^{2}{ }_{3}\right) / a_{0}{ }^{3} \\
& +\left(7 a_{1} a_{6}+7 a_{2} a_{5}+7 a_{3} a_{4}\right) / a_{0}^{2}-7 a_{7} / a_{0} \text {, } \\
& S_{8}=a_{1}^{8} / a_{0}^{8}-8 a_{1}^{6} a_{2} / a_{0}{ }^{7}+\left(8 a_{1}^{5} a_{3}+20 a_{1}^{4} a_{2}^{2}\right) / a_{0}^{6} \\
& -\left(8 a_{1}^{4} a_{4}+32 a_{1}^{3} a_{2} a_{3}+16 a_{1}^{2} a^{3}{ }_{2}\right) / a_{0}^{5} \\
& +\left(8 a_{1}^{3} a_{5}+24 a_{1}^{2} a_{2} a_{4}+12 a_{1}^{2} a_{3}{ }^{2}+24 a_{1} a_{2}{ }^{2} a_{3}+2 a_{2}^{4}\right) / a_{0}^{4} \\
& -\left(8 a_{1}^{2} a_{6}+16 a_{1} a_{2} a_{5}+16 a_{1} a_{3} a_{4}+8 a_{2}^{2} a_{4}+8 a_{2} a_{3}^{2}\right) / a_{0}^{3} \\
& +\left(8 a_{1} a_{7}+8 a_{2} a_{6}+8 a_{3} a_{5}+4 a_{4}^{2}\right) / a_{0}^{2}-8 a_{8} / a_{0} \text {, } \\
& S_{9}=-a_{1}{ }^{9} / a_{0}{ }^{9}+9 a_{1}{ }^{7} a_{2} / a_{0}{ }^{8}-\left(9 a_{1}{ }^{6} a_{3}+27 a_{1}{ }^{5} a_{2}{ }^{2}\right) / a_{0}{ }^{7} \\
& +\left(9 a_{1}{ }^{5} a_{4}+45 a_{1}{ }^{4} a_{2} a_{3}+30 a_{1}^{3} a_{2}{ }^{3}\right) / a_{0}{ }^{6} \\
& -\left(9 a_{1}^{4} a_{5}+36 a_{1}^{3} a_{2} a_{4}+18 a_{1}^{3} a_{3}^{2}+54 a_{1}^{2} a_{2}^{2} a_{3}+9 a_{1} a_{2}^{4}\right) / a_{0}^{5} \\
& +\left(9 a_{1}^{3} a_{6}+27 a_{1}^{2} a_{2} a_{5}+27 a_{1}^{2} a_{3} a_{4}+27 a_{1} a_{2} a_{3}^{2}+9 a_{2}^{3} a_{3}\right) / a_{0}^{4} \\
& -\left(9 a_{1}^{2} a_{7}+18 a_{1} a_{2} a_{6}+18 a_{1} a_{3} a_{5}+9 a_{1} a_{4}{ }^{2}+9 a_{2}^{2} a_{5}+18 a_{2} a_{3} a_{4}+3 a_{3}^{3}\right) / a_{0}{ }^{3} \\
& +\left(9 a_{1} a_{8}+9 a_{2} a_{7}+9 a_{3} a_{6}+9 a_{4} a_{5}\right) / a_{0}^{2}-9 a_{9} / a_{0} \text {, } \\
& S_{10}=a_{1}{ }^{10} / a_{0}{ }^{10}-10 a_{1}{ }^{8} a_{2} / a_{0}{ }^{9}+\left(10 a_{1}{ }^{7} a_{3}+35 a_{1}{ }^{6} a_{2}{ }^{2}\right) / a_{0}{ }^{8} \\
& -\left(10 a_{1}^{6} a_{4}+60 a_{1}{ }^{5} a_{2} a_{3}+50 a_{1}^{4} a_{2}^{3}\right) / a_{0}{ }^{7}+\left(10 a_{1}{ }^{5} a_{5}+50 a_{1}^{4} a_{2} a_{4}\right. \\
& \left.+25 a_{1}^{4} a_{3}^{2}+100 a_{1}^{3} a_{2}^{2} a_{3}+25 a_{1}^{2} a_{2}^{4}\right) / a_{0}^{6}-\left(10 a_{1}^{4} a_{6}+40 a_{1}^{3} a_{2} a_{5}\right. \\
& \left.+40 a_{1}^{3} a_{3} a_{4}+60 a_{1}^{2} a_{2}^{2} a_{4}+60 a_{1}^{2} a_{3}^{2} a_{2}+40 a_{2}^{3} a_{3} a_{1}+2 a_{2}{ }^{5}\right) / a_{0}{ }^{5} \\
& +\left(10 a_{1}^{3} a_{7}+30 a_{1}^{2} a_{2} a_{6}+30 a_{1}^{2} a_{3} a_{5}+15 a_{1}^{2} a_{4}^{2}+30 a_{1} a_{2}^{2} a_{5}\right. \\
& \left.+60 a_{1} a_{2} a_{3} a_{4}+10 a_{2}^{3} a_{4}+15 a_{2}^{2} a_{3}^{2}+10 a_{1} a_{3}{ }^{3}\right) / a_{0}^{4} \\
& -\left(10 a_{1}^{2} a_{8}+20 a_{1} a_{2} a_{7}+20 a_{1} a_{3} a_{6}+20 a_{1} a_{4} a_{5}+20 a_{2} a_{3} a_{5}\right. \\
& \left.+10 a_{2} a_{4}^{2}+10 a_{2}^{2} a_{6}+10 a_{3}^{2} a_{4}\right) / a_{0}^{3}+\left(10 a_{1} a_{9}+10 a_{2} a_{8}\right. \\
& \left.+10 a_{3} a_{7}+10 a_{4} a_{6}+5 a_{5}^{2}\right) / a_{0}^{2}-10 a_{10} / a_{0} \text {, } \\
& S_{11}=-a_{1}^{11} / a_{0}{ }^{11}+11 a_{1}{ }^{9} a_{2} / a_{0}{ }^{10}-\left(11 a_{1}{ }^{8} a_{3}+44 a_{1}{ }^{7} a_{2}{ }^{2}\right) / a_{0}{ }^{9} \\
& +\left(11 a_{1}{ }^{7} a_{4}+77 a_{1}{ }^{6} a_{2} a_{3}+77 a_{1}{ }^{6} a_{2}{ }^{3}\right) / a_{0}{ }^{8}-\left(11 a_{1}{ }^{6} a_{5}+66 a_{1}{ }^{5} a_{2} a_{4}\right. \\
& \left.+33 a_{1}{ }^{5} a^{2}{ }_{3}+165 a_{1}{ }^{4} a_{2}{ }^{2} a_{3}+55 a_{1}{ }^{3} a_{2}{ }^{4}\right) / a_{0}{ }^{7}+\left(11 a_{1}{ }^{5} a_{6}+55 a_{1}^{4} a_{2} a_{5}\right. \\
& \left.+55 a_{1}^{4} a_{3} a_{4}+110 a_{1}^{2} a_{2}^{3} a_{3}+110 a_{1}^{3} a_{2}^{2} a_{4}+110 a_{1}^{3} a_{2} a_{3}{ }^{2}+11 a_{1} a_{2}{ }^{5}\right) / a_{0}{ }^{6} \\
& -\left(11 a_{1}^{4} a_{7}+44 a_{1}^{3} a_{2} a_{6}+44 a_{1}^{3} a_{3} a_{5}+22 a_{1}^{3} a_{4}{ }^{2}+66 a_{1}^{2} a_{2}{ }^{2} a_{5}\right. \\
& \left.+132 a_{1}^{2} a_{2} a_{3} a_{4}+44 a_{1} a_{2}^{3} a_{4}+66 a_{1} a_{2}^{2} a_{3}^{2}+11 a_{2}^{4} a_{3}+22 a_{1}^{2} a_{3}^{3}\right) / a_{0}^{5} \\
& +\left(11 a_{1}^{3} a_{8}+33 a_{1}^{2} a_{2} a_{7}+33 a_{1}^{2} a_{3} a_{6}+33 a_{1}^{2} a_{4} a_{5}+33 a_{1} a_{2}^{2} a_{6}\right. \\
& \left.+66 a_{1} a_{2} a_{3} a_{5}+33 a_{1} a_{2} a_{4}{ }^{2}+33 a_{1} a_{3}{ }^{2} a_{4}+11 a_{2}{ }^{3} a_{5}+33 a_{2}{ }^{2} a_{3} a_{4}+11 a_{3}{ }^{3} a_{2}\right) / a_{0}{ }^{4} \\
& -\left(11 a_{1}^{2} a_{9}+22 a_{1} a_{2} a_{8}+22 a_{1} a_{3} a_{7}+22 a_{1} a_{4} a_{6}+11 a_{1} a_{5}^{2}+11 a_{2}^{2} a_{7}\right. \\
& \left.+22 a_{2} a_{3} a_{6}+22 a_{2} a_{4} a_{5}+11 a_{3} a_{4}^{2}+11 a_{3}^{2} a_{5}\right) / a_{0}^{3}+\left(11 a_{1} a_{10}+11 a_{2} a_{9}\right. \\
& \left.+11 a_{3} a_{8}+11 a_{4} a_{7}+11 a_{5} a_{6}\right) / a_{0}^{2}-11 a_{11} / a_{0} \text {. }
\end{aligned}
$$


combination of $a$ 's yields the numerical coefficient for that combination. For example, in the summation for $S_{11}$ given in Table $2,1 / a_{0}{ }^{4}$ has as the $a_{1}{ }^{k-1} a_{n-k+1}$ coefficient $a_{1}{ }^{3} a_{8}$ which, in turn, has the numerical coefficient 11 . The $a_{1}^{2} a_{3} a_{6}$ combination has $2 ! \times 1 ! \times 1$ ! as the product of the factorials of the exponents. If $11 \times 3 ! \times 1$ ! is divided by $2 ! \times 1 ! \times 1$ !, the result is 33 which is the numerical coefficient of the $a_{1}^{2} a_{3} a_{6}$ combination.

Construction of Table 1. Table 1 shows the number of coefficients of $-1 / a_{0}$ as the extreme right-hand entry corresponding to a given $n$. The next entry to the left is the number of coefficients for $+1 / a_{0}^{2}$, etc. The pattern for the number of $-1 / a_{0}$ and $+1 / a_{0}{ }^{2}$ coefficients is obvious. It should also be noted that entries to the left of the stepped division remain fixed for $n$ and $k$. The entries in a column for $k=(n-h)$, where $h=0,1,2$, etc., are formed from cumulative sums, starting from the right, of the entries in the row for $n=h$. For example, for $n=6, h=5$, and $k=1$, the entry is 1 . This entry is equal to the first 1 on the right in the row for $n=5$. For $n=7, h=5$, and $k=2$, the entry is 3 . This entry is equal to $(1+2)$ which is the sum of the first two right-hand entries of the row for $n=5$. A column is formed in this fashion until the sum of the entries in the row (in this case, the fifth) is arrived at, after which the entries in the column remain fixed at this value. The fixed values are those to the left of the stepped division, as explained above.

Summary. The method of finding $S_{n}$ may be summarized briefly as follows. First, obtain the number of coefficients of each $(-1)^{k} / a_{0}{ }^{k}$ from Table 1 or an extension thereof. Second, by some systematic procedure obtain as many different combinations of the $a$ 's as there are coefficients specified by Table 1, making certain that the weight of each combination equals $n$ and the degree equals $k$. Third, find $n \times(k-1)$ ! divided by the product of the factorials of the exponents to obtain the numerical value of a combination. A listing of $S_{n}$ from $n=1$ to $n=11$ is given in Table 2 .

Georgia Institute of Technology

Atlanta, Georgia

1. E. P. AdAMs, Smithsonian Mathematical Formulae and Tables of Elliptic Functions, Smithsonian Institution, Washington, 1947, p. 2-3.

2. G. ChRYstal, Textbook of Algebra, Vol. 1, Chelsea Publishing Co., New York, 1952, p. 434.

3. G. Chrystal, Textbook of Algebra, Vol. 2, Chelsea Publishing Co., New York, 1952, p. 558.

\title{
An Alternating Direction Method for Solving The Biharmonic Equation
}

\author{
By S. D. Conte and R. T. Dames
}

1. Introduction. The biharmonic operator, defined by

$$
\Delta \Delta=\frac{\partial^{4}}{\partial x^{4}}+2 \frac{\partial^{4}}{\partial x^{2} \partial y^{2}}+\frac{\partial^{4}}{\partial y^{4}},
$$

plays a role in the classical theory of plates similar to that of the Laplace operator in potential theory. In particular, the biharmonic equation, $\Delta \Delta W=0$, together

Received 4 October 1957. 